\title{
A case report of acute severe paraquat poisoning and long-term follow-up
}

\author{
GUANGCAI YU* , BAOTIAN KAN* , XIANGDONG JIAN，JIERU WANG，JING SUN and CHENGZHEN SONG \\ Department of Poisoning and Occupational Diseases, Qilu Hospital of Shandong University, \\ Jinan, Shandong 250012, P.R. China
}

Received December 22, 2013; Accepted May 9, 2014

DOI: 10.3892/etm.2014.1727

\begin{abstract}
In the present study, the successful management of severe paraquat (PQ) poisoning with multiple organ dysfunction syndrome is described. A 42-year-old female ingested $>100 \mathrm{ml}$ PQ (20\% weight/volume) in an attempted suicide. After $22 \mathrm{~h}$ the patient was admitted to hospital with serious liver, kidney and lung damage. Comprehensive therapy that maximized poison elimination was administered, along with appropriate glucocorticoids and medication for anticoagulation and protection of the liver and kidney. The patient was successfully treated and recovered after 40 days. However, pulmonary damage was aggravated when the glucocorticoid treatment was stopped after 2 months; the lungs recovered again following systematic therapy. Subsequent to a 8-month follow-up, the patient was able to look after herself in her daily life. To the best of our knowledge, successful treatment following severe PQ poisoning is rare.
\end{abstract}

\section{Introduction}

Paraquat (N,N'-dimethyl-4,4'-bipyridinium dichloride; PQ), an effective herbicide that has favorable environmental characteristics, as well as being cost-effective, was first synthesized in 1882 and has been used as an herbicide since 1955 (1). PQ is an important herbicide used in agriculture; however, thousands of individuals succumb due to PQ intoxication every year in the developing world. PQ is a highly toxic compound and the fatality rate of PQ ranges between 60 and $80 \%$ (2) due to the lack of a specific antidote. A PQ dose of $30 \mathrm{mg} / \mathrm{kg}$ may be fatal, which is equivalent to $8-10 \mathrm{ml}$ of the $20 \%$ solution sold commercially (3). PQ has been shown to cause significant damage to organs, including the lung, liver,

Correspondence to: Professor Xiangdong Jian, Department of Poisoning and Occupational Diseases, Qilu Hospital of Shandong University, 107 Wenhuaxi Road, Jinan, Shandong 250012, P.R. China E-mail: jianxiangdong@sdu.edu.cn

${ }^{*}$ Contributed equally

Key words: acute paraquat poisoning, treatment, long-term follow kidneys and myocardium, with the highest concentration of PQ found in the lungs (4). The prognosis of patients with multiple organ dysfunction syndrome (MODS) caused by fulminant poisoning ( $>40 \mathrm{mg}$ PQ ion per $\mathrm{kg}$ of body weight) is extremely dangerous and patients may succumb within hours to a few days following ingestion $(5,6)$.

China is an agricultural country and PQ is used extensively. Since the first reported case of PQ poisoning, studies have focused on the mechanism and effects of combination therapies and various agents $(7,8)$. Furthermore, strategies for the management of PQ poisoning have focused on the modification of the toxicokinetics of the poison by either decreasing its absorption or enhancing its elimination (9); however, studies on comprehensive strategies are rare. We previously identified a comprehensive treatment strategy against PQ poisoning, termed the Qilu scheme (10). In the present study, a case of MODS caused by severe PQ poisoning was treated using multi-target comprehensive therapy. Furthermore, the present study indicates the potential and feasibility of the therapy for the treatment of PQ poisoning. Informed consent was obtained from the patient. The study was approved by the ethics committee of the Qilu Hospital, Shandong University (Jinan, China).

\section{Case report}

A 42-year-old female, weighing $\sim 60 \mathrm{~kg}$, ingested $>100 \mathrm{ml}$ PQ (20\% weight/volume) in an attempted suicide at 12:50 p.m. on September 13, 2012. The patient experienced nausea, vomiting and discomfort immediately following PQ ingestion and was sent to a county hospital 20 min later. A gastric lavage was immediately performed and kaolin (30 g) and mannitol infusion $(250 \mathrm{ml})$ was administered within $1 \mathrm{~h}$ following ingestion. Hemoperfusion therapy was prescribed for $2 \mathrm{~h}$. The dose for methylprednisolone was $1,000 \mathrm{mg} /$ day for the first day. Due to the severity of the condition, relatives brought the patient to the Department of Poisoning and Occupational Diseases, Qilu Hospital of Shandong University at 10:55 a.m. on December 14, 2012. On admission, the patient's vital signs were normal, with a body temperature $36.6^{\circ} \mathrm{C}$, pulse 89 beats/min, respiratory rate 19 breaths/min, blood pressure $125 / 80 \mathrm{mmHg}$ and blood oxygen saturation $98 \%$. Physical examination was normal, with the exception of painful erosions in the oral cavity. Based on the typical 
Table I. Changes in the main indicators of blood, urine, liver and kidney function test results for the patient 2, 6, 14, 43, 71, 127 and 221 days following ingestion.

\begin{tabular}{|c|c|c|c|c|c|c|c|c|c|c|c|c|c|}
\hline Date & $\begin{array}{l}\text { ALT }^{*} \\
(\mathrm{U} / \mathrm{l})\end{array}$ & $\begin{array}{l}\text { AST }^{*} \\
\text { (U/l) }\end{array}$ & $\begin{array}{c}\mathrm{GGT}^{*} \\
(\mathrm{U} / \mathrm{l})\end{array}$ & $\begin{array}{c}\mathrm{Cr} \\
(\mu \mathrm{mol} / \mathrm{l})\end{array}$ & $\begin{array}{c}\text { BUN } \\
(\mathrm{mmol} / \mathrm{l})\end{array}$ & $\begin{array}{c}\mathrm{CK}^{\mathrm{CMB}}{ }^{*} \\
(\mathrm{ng} / \mathrm{ml})\end{array}$ & $\begin{array}{l}\mathrm{CK}^{*} \\
\text { (U/l) }\end{array}$ & $\begin{array}{c}\mathrm{DDi}^{*} \\
(\mu \mathrm{g} / \mathrm{ml})\end{array}$ & $\begin{array}{c}\text { WBC } \\
\left(10^{9} / 1\right)\end{array}$ & $\begin{array}{c}\text { HGB } \\
(\mathrm{g} / \mathrm{l})\end{array}$ & $\begin{array}{l}\text { PLT } \\
\left(10^{9} / 1\right)\end{array}$ & $\begin{array}{c}\text { ESR } \\
(\mathrm{mm} / \mathrm{h})\end{array}$ & PRO \\
\hline Day 2 & 219 & 212 & 108 & 241 & 13.3 & 4.2 & 410 & 0.79 & 21.05 & 140 & 115 & 16 & $1+$ \\
\hline Day 6 & 815 & 304 & 797 & 274 & 23.9 & 2.2 & 117 & 0.85 & 12.79 & 132 & 162 & 44 & $1+$ \\
\hline Day 14 & 53 & 39 & 316 & 53 & 5.4 & 5.2 & 75 & 0.70 & 20.76 & 115 & 369 & 69 & $1+$ \\
\hline Day 43 & 171 & 82 & 610 & 41 & 5.7 & 2.5 & 29 & 0.16 & 9.08 & 118 & 199 & 40 & - \\
\hline Day 71 & 27 & 24 & 156 & 40 & 1.8 & 1.5 & 28 & 0.64 & 5.95 & 135 & 220 & 27 & +- \\
\hline Day 127 & 29 & 20 & 58 & 57 & 4.4 & 2.0 & 31 & 0.54 & 11.39 & 128 & 236 & 13 & - \\
\hline Day 221 & 23 & 22 & 49 & 60 & 2.6 & 0.8 & 64 & 0.30 & 5.74 & 125 & 207 & 2 & - \\
\hline
\end{tabular}

"Reference values: ALT, AST (0-40 U/l); CK-MB (0.3-4.0 ng/ml); CK (26-140 U/l); DDi (0-0.5 $\mu$ g/ml); GGT (3-50 U/1). Blood biochemistry was tested using a Roche cobas ${ }^{\circledR} 8000$ automatic biochemical analyzer; blood routine, urine routine and blood sedimentation was tested using the Sysmex XE-2100 automatic biochemical analyzer; DDi was tested using Beckman Kurt's ACL TOP 700 automatic blood coagulation analyzer. ALT, alanine aminotransferase; AST, aspartate aminotransferase; GGT, glutathione-S-transferase; CR, creatinine; BUN, blood urea nitrogen; CK-MB, creatine kinase MB isoenzyme; DDi, D-dimer; WBC, white blood cell; HGB, hemoglobin; PLT, platelet; ESR, erythrocyte sedimentation rate; PRO, urine protein. The values $1+,-$ and +- indicate positive and suspicious positive.

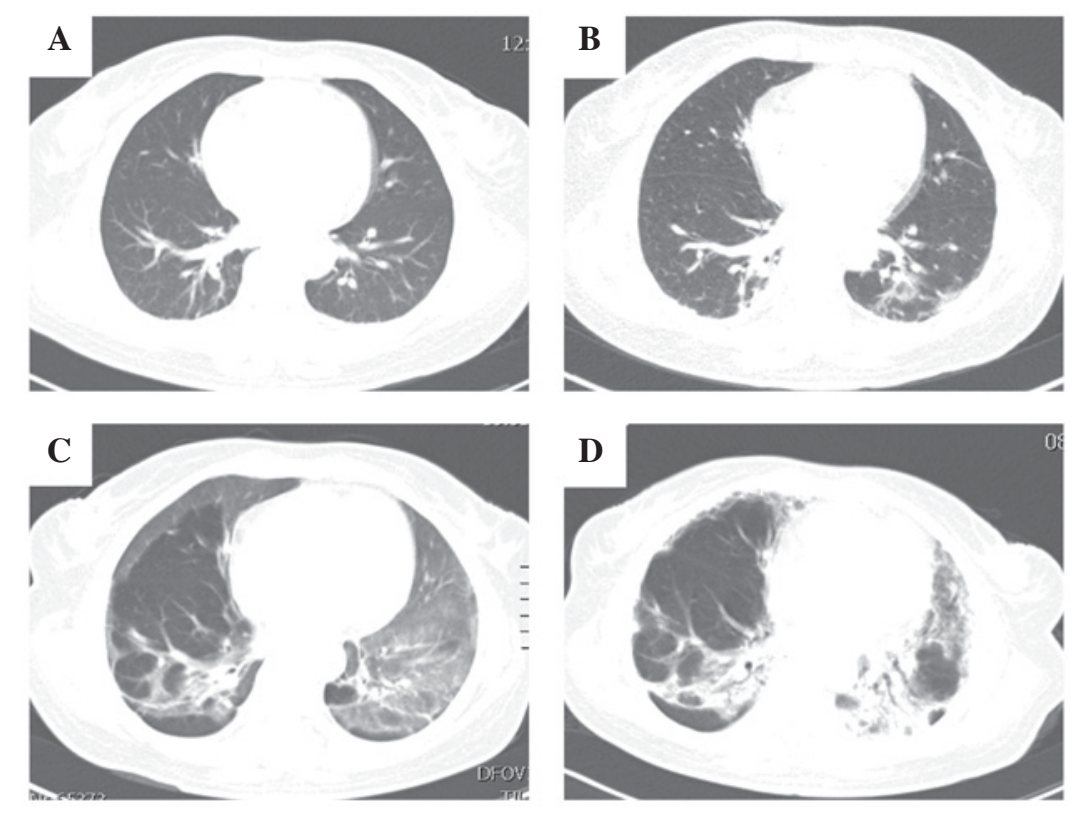

Figure 1. Lung CT scans at different time-points following ingestion of paraquat. (A) Initial CT scan (2 days following ingestion) showing increased lung markings and pleural thickening, with small inflammatory patches observed around the pleura. (B) CT scan 5 days following ingestion. Cotton-wool spots and cloudy high density are visible, primarily in the left lung. (C) A follow up CT scan (15 days following ingestion) demonstrating showing diffuse patchy shadows and ground glass appearance, observed primarily in the left lung. (D) At 24 days following ingestion, the ground-glass attenuation had decreased markedly, fibrotic changes were dominant in the lung. CT, computed tomography.

poison-associated symptoms in the mouth and the gastrointestinal tract, computed tomography (CT) manifestations, significantly abnormal results in the main laboratory test results and the information provided by the patient and her relatives on admission, the patient was diagnosed with severe acute PQ poisoning.

Following admission to Qilu Hospital, the patient was subjected to complete gastrointestinal decontamination using activated carbon, Smecta and mannitol in order to thoroughly remove the pesticide residue from the gastrointestinal tract. Furthermore, other treatments such as an intravenous drip of high doses of glucocorticoids, anticoagulants, anti-free radical, liver- and kidney-protecting and water and electrolyte balance-maintaining agents, and treatment with Chinese herbs (including Xuebijing, Cordyceps and depside salt from Salvia miltiorrhiza) were administered to the patient. This was termed the Qilu therapeutic schedule. The dose for glucocorticoids was $800 \mathrm{mg} /$ day for the first 4 days and then reduced to $40-80 \mathrm{mg} / \mathrm{day}$. The patient's condition was markedly improved following the comprehensive therapy. Low doses of prednisone were maintained after 3-6 weeks. After 2 months the patient stopped taking glucocorticoids; 


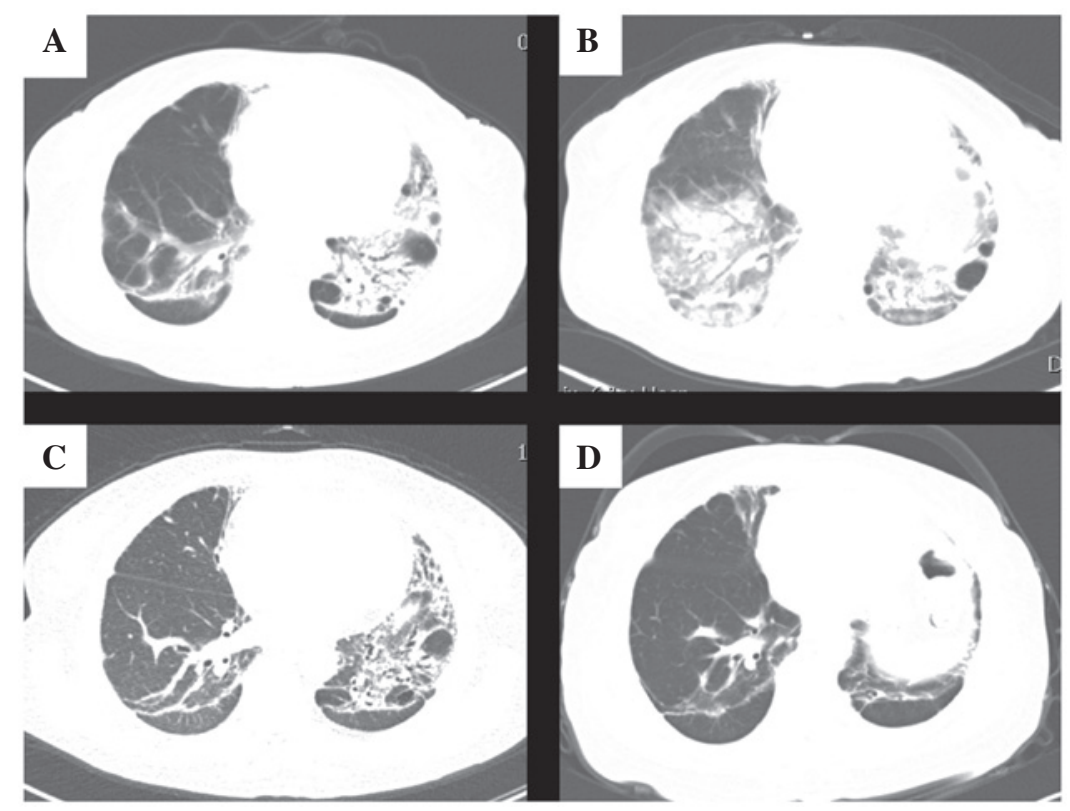

Figure 2. Lung CT scans at different time-points following ingestion of paraquat. (A) At 44 days following ingestion, the lung fibrosis had decreased. (B) At 75 days post-ingestion the lung damage was aggravated. Larger lesions with air and fluid bronchograms, surrounded by a wide range of patchy lesions were observed. (C) A follow up CT scan after 148 days showed that the lung damage had decreased markedly once the treatment was resumed. (D) A CT of the lung 236 days following paraquat exposure revealed localized fibrosis was improved. CT, computed tomography.

however, the pulmonary damage became aggravated and recovered again subsequent to treatment with glucocorticoid and traditional Chinese medicines.

Following multi-target comprehensive therapy, the dynamic changes in the lung CT scans are shown in Fig. 1 and 2, and the main laboratory test results are shown in Table I. The liver and kidney functions were seriously damaged 2 days following ingestion and the most serious damage was observed 6 days subsequent to PQ poisoning. However, the liver and kidney functions gradually recovered 2 weeks later (Table I). The initial CT scan showed that there was a certain degree of lung injury that occurred after 2 days (Fig. 1A) and gradually accelerated over the following 3 days (Fig. 1B). Serious lung injury occurred 15 days later (Fig. 1C); however, it improved gradually during the 6 weeks of treatment (Fig. 1D and Fig. 2A). Treatment was ceased gradually; however, after 2 months the lung injury re-occurred (Fig. 2B) accompanied by marked liver dysfunction. Treatment was then resumed and lung function improved again (Fig. 2C), and the liver function gradually returned to normal. Eight months following ingestion, the patient's lung function was markedly improved (Fig. 2D). The pulmonary function test indicated moderate restrictive ventilation dysfunction and a mild decline of diffuse lung function with the forced vital capacity, maximum vital capacity, maximum ventilatory volume and carbon monoxide diffusion capacity accounting for 53.2, 52.1, 67.3 and $54.1 \%$ of the expected values, respectively. The patient continues to be followed up.

\section{Discussion}

PQ is a non-selective herbicide that has been widely used in agriculture since the 1960s (11). Although it has been found to be safe for occupational use, $\mathrm{PQ}$ poisoning has been observed in patients who ingest the pesticide either accidentally or inten- tionally in an attempt to commit suicide. PQ is banned or rarely used in the developed world; however, in developing countries $\mathrm{PQ}$ continues to be used and PQ poisoning remains a major cause of mortality among patients with acute poisoning (12). During acute PQ poisoning, the pulmonary concentrations of PQ may be higher than the plasma concentration (13). Therefore, the primary cause of mortality in PQ poisoning is respiratory failure due to oxidative damage to the alveolar epithelium with subsequent obliterating fibrosis (14). In addition to lung damage, $\mathrm{PQ}$ ingestion has been shown to injure other organs, but to a lesser extent. Severe PQ poisoning is characterized by multi-organ involvement, primarily affecting the lungs, kidneys, liver, myocardium and adrenal cortex (15). Therefore, urgent gastric lavage is required to reduce the absorption of PQ. Extracorporeal elimination, for example hemoperfusion (HP), is an effective measure that has previously been used clinically (11). The majority of studies have focused on the association between HP and the overall prognosis of PQ poisoning. However, the results from these studies are discouraging and controversial opinions have arisen on the therapeutic effects of HP in PQ intoxication treatment $(16,17)$. The prognosis for patients with severe PQ poisoning, complicated with multiple organ damage, is extremely poor. Despite numerous studies into the mechanism of toxicity and the potential therapeutic treatments for PQ poisoning, at present no specific therapy has been shown to affect the outcome in controlled clinical studies (12).

In the present case study, a patient with PQ poisoning complicated with MODS was successfully treated using multi-target comprehensive therapy. The patient ingested a high dose of PQ, resulting in severe liver and kidney damage. Gastrointestinal procedures were performed to prevent further absorption of PQ residue, accompanied by glucocorticoid treatment to reduce cell absorption, promote the 
elimination of PQ and control lung inflammation lesions. The mechanism of PQ toxicity has been previously investigated by Dinis-Oliveira et al (18). In the present study, anticoagulant and antioxidant treatments, as well as Chinese medicine, were administered to the patient to prevent oxidative damage and to protect other organs against injury. Pulmonary lesions gradually developed into pulmonary fibrosis; therefore, the patient was given the immunosuppressant cyclophosphamide. The patient's condition was aggravated following the cessation of the treatment. The lung injury was improved once treatment with glucocorticoids was resumed, indicating that glucocorticoids may have an important role in the treatment of pulmonary fibrosis caused by PQ poisoning. The patient was cured and discharged from hospital. However, further studies are required to determine an effective treatment against PQ poisoning.

\section{Acknowledgements}

This study was supported by the Natural Science Foundation of Shandong Province (no. Y2008C123).

\section{References}

1. Yang CJ, Lin JL, Lin-Tan DT, Weng CH, et al: Spectrum of toxic hepatitis following intentional paraquat ingestion: analysis of 187 cases. Liver Int 32: 1400-1406, 2012.

2. Weng CH, Hu CC, Lin JL, Lin-Tan DT, et al: Sequential organ failure assessment score can predict mortality in patients with paraquat intoxication. PLoS One 7: e51743, 2012.

3. Yoon SC: Clinical outcome of paraquat poisoning. Korean $\mathrm{J}$ Intern Med 24: 93-94, 2009.

4. Yen TH, Lin JL, Lin-Tan DT, Hsu CW, et al: Spectrum of corrosive esophageal injury after intentional paraquat ingestion. Am J Emerg Med 28: 728-733, 2010.

5. Agarwal R, Srinivas R, Aggarwal AN and Gupta D: Immunosuppressive therapy in lung injury due to paraquat poisoning: a meta-analysis. Singapore Med J 48: 1000-1005, 2007.
6. Sabzghabaee AM, Eizadi-Mood N, Montazeri K, Yaraghi A and Golabi M: Fatality in paraquat poisoning. Singapore Med J 51: 496-500, 2010.

7. Lin JL, Lin-Tan DT, Chen KH, Huang WH, et al: Improved survival in severe paraquat poisoning with repeated pulse therapy of cyclophosphamide and steroids. Intensive Care Med 37: 1006-1013, 2011.

8. Sun ML, Ma DH, Liu M and Yu YX: Successful treatment of paraquat poisoning by Xuebijing, an injection concocted from multiple Chinese medicinal herbs: a case report. J Altern Complement Med 15: 1375-1378, 2009.

9. Dinis-Oliveira RJ, Sarmento A, Reis P, Amaro A, et al: Acute paraquat poisoning: report of a survival case following intake of a potential lethal dose. Pediatr Emerg Care 22: 537-540, 2006.

10. Jian X, Zhang H, Sui H, Guo G, et al: Qilu Scheme of paraquat poisoning treatment (2014). Chinese Journal of Industrial Medicine (Chinese) 27: 119-121, 2014 (In Chinese).

11. Zhang Z, Jian X, Zhang W, Wang J and Zhou Q: Using bosentan to treat paraquat poisoning-induced acute lung injury in rats. PLoS One 8: e75943, 2013.

12. Wilks MF, Fernando R, Ariyananda PL, Eddleston M, et al: Improvement in survival after paraquat ingestion following introduction of a new formulation in Sri Lanka. PLoS Med 5: e49, 2008.

13. Rose MS, Smith LL and Wyatt I: Evidence for energy-dependent accumulation of paraquat into rat lung. Nature 252, 314-315, 1974.

14. Suntres ZE: Role of antioxidants in paraquat toxicity. Toxicology 180: 65-77, 2002.

15. Yeo CD, Kim JW, Kim YO, Yoon SA, et al: The role of pentraxin-3 as a prognostic biomarker in paraquat poisoning. Toxicol Lett 212: 157-160, 2012.

16. Castro R, Prata C, Oliveira L,Carvalho MJ, et al: Paraquat intoxication and hemocarboperfusion. Acta Med Port 18: 423-431, 2005 (In Portuguese).

17. Hampson EC and Pond SM: Failure of haemoperfusion and haemodialysis to prevent death in paraquat poisoning. Med Toxicol Adverse Drug Exp 3: 64-71, 1988.

18. Dinis-Oliveira RJ, Duarte JA, Sánchez-Navarro A, Remião F, et al: Paraquat poisonings: mechanisms of lung toxicity, clinical features, and treatment. Crit Rev Toxicol 38: 13-71, 2008. 